\title{
Reflexes of Proto-Austronesia into Buru Language: Preliminary Step of Testing Collins Hypothesis (1981)
}

\author{
Sumarlam \\ Postgraduate Doctoral Program of Linguistics \\ Universitas Sebelas Maret (UNS) \\ Surakarta, Indonesia \\ sumarlamwd@gmail.com \\ Djatmika \\ Postgraduate Doctoral Program of Linguistics \\ Universitas Sebelas Maret (UNS) \\ Surakarta, Indonesia \\ djatmika@staff.uns.ac.id
}

\author{
Dwi Purnanto \\ Postgraduate Doctoral Program of Linguistics \\ UniversitasSebelasMaret (UNS) \\ Surakarta, Indonesia \\ dwi.purnanto@yahoo.com \\ Burhanuddin \\ Postgraduate Doctoral Program of Linguistics and \\ Departement of Language and Art \\ Universitas Sebelas Maret (UNS) and Universitas Mataram \\ (Unram) \\ Surakarta and Mataram, Indonesia \\ burhanuddinali.fkipunram@yahoo.co.id
}

\begin{abstract}
This paper intends to explain two issues, the Proto-Austronesian reflex (PAN) into Buru language (Br) and its correspondence with Collins's (1981) view. A PAN top-down approach reconstructed by Blust and Trussel (2015) is employed to see the realization in Buru. The data that have been collected using direct interview are 200 basic vocabulary revised by Blust (1980) and approximately 800 cultural vocabularies. The results identify that (1) Buru speech sounds that tend to innovate from PAN, ie * $k$, * b, * $\mathbf{q}, * \mathbf{h}, * \mathbf{S}, * \mathbf{C}, * \mathbf{d}, * \mathbf{z}, *$ ay, and * uy; (2) Buru speech sounds that tend to retain, ie * p, * t, * $\mathbf{g},{ }^{*} \mathbf{y},{ }^{*} \mathbf{m},{ }^{*} \mathbf{w},{ }^{*} \mathbf{s},{ }^{*} \mathbf{r},{ }^{*} \mathbf{l},{ }^{*} \mathbf{a},{ }^{*} \mathbf{u}, * \mathbf{i}, *$ $\partial$; (3) not found PAN * $t$ that change to / f / in Buru as proof of unity with Sula and Taliabo as well as proof of separation with Ambelau language. PAN * $t$ in the initial and middle position only changes to / $t /, / \mathrm{n} /$, and $/ \varnothing /$.
\end{abstract}

Keywords—reflex, Proto-Austronesian, Buru language, Collins hypothesis (1981)

\section{INTRODUCTION}

In 1981, James Collins conducted a preliminary study of the language group he called the West Central Maluku. This group consists of Ambelau ( $\mathrm{Ab}$ ), Buru (Br), Sula ( $\mathrm{Sl})$, and Taliabo $(\mathrm{Tb})$. in linguistic-historical view, these four languages have one origin, then split into two, Ambelau and Proto-Buru-Sula-Taliabo. Proto-Buru-Sula-Taliabo then split into two, namely Buru and Proto-Sula-Taliabo, and finally Sula and Taliabo. The Collins (1981) study was conducted using a top-down approach, a comprehensive innovation method examining the Proto-Austronesian (PAN) reflexes into the four languages as well as three isolates in the Middle East Moluccas. Since the study is preliminary, it needs to be verified and enriched with linguistic evidence.

Sumarlam et al (2017) has identified the pattern of PAN change into Ambelau language to observe the truth of the description Collins (1981) about the language. The same pattern is applied, not only in Ambelau but also in three other languages, Buru, Sula, and Taliabo. Therefore, this paper identifies the PAN reflex into Buru language and sees its compatibility with Collins's (1981) view.

Collins (1981) at least suggested five types of PAN reflexes into Buru language. Firstly, the PAN $* \mathrm{t}$ in the initial position (\#-) and the vowel (\# v-v \#) change into / $\mathrm{f} / \mathrm{in}$ Buru (also in Sula and Taliabo). Secondly, PAN: * k / \# - and \# v-v \# in Buru have retention. Thirdly, PAN: * mb changes to / b / in Buru (also Sula and Taliabo). Fourth, there are merger of PAN: * $y$ and $* \eta \mathrm{k}$ into /g /. Moreover, the five merger of PAN: $* \mathrm{k}$ and $* \mathrm{p}$ at the end position changed to $/ \mathrm{t} /$. Whether the linguistic evidence proposed by Collins (1981) is sufficient, this paper intends to explain it. Therefore, this paper aims at explaining two things, the PAN reflex into Buru language and its compatibility with Collins's (1981) view.

\section{METHOD}

To explain these two aspects, data collection, data analysis, and presentation of data analysis results have been conducted. Field data, collected using interview technique, consist of 200 basic vocabularies and 800 cultural vocabularies in Buru language. The Proto-Austronesian data were collected using literature method in the form of an Austronesian Comparative Dictionary 
compiled by Blust and Trussel (2015). The collected data were then analyzed using a top-down approach, an innovation method viewing the realization of PAN into Buru language. Furthermore, the analyzed data were conceptualized and presented using nonformal methods (using regular words) and formal methods (using images, symbols, graphs, tables, etc.).

\section{FINDING AND DISCUSSION}

In order to systematically describe the PAN reflex into Buru language, the number and type of PAN phonemes will be presented. According to Trussell and Blust (2015), PAN phoneme consists of 32 phonemes including 24 consonants, (/p, t, C, c, $\mathrm{k}, \mathrm{P}, \mathrm{b}, \mathrm{d}, \mathrm{z}, \mathrm{j}, \mathrm{g}, \mathrm{m}, \mathrm{n}, \mathrm{n}, \mathrm{y}, \mathrm{s}, \mathrm{S}, \mathrm{h}, \mathrm{l}, \mathrm{r}, \mathrm{R}, \mathrm{y}$, and $\mathrm{w} /$ ), four vowels (/ i, u, ə, and a /), and four diphthongs (-aw, -ay, -uy, and -iy). Sumarlam et al (2017) identifies that Buru language has 19 consonant phonemes, i.e. / p, t, c, k, ?, b, d, j, g, m, n, n, y, s, h, l, r, y, and $\mathrm{w} /$ and eight vowels (/ i, u, e, , , $\varepsilon, \mathrm{o}, \mathrm{o}$, and a /).

A. $P A N * p$

PAN * $\mathrm{p}$ at initial and intervocalic positions in Buru language are retained and regular

$\begin{array}{llll}\text { Gloss } & \text { PAN } & \text { Buru } & \text { Rule } \\ \text { navel } & \text { *pujok } & \text { puscn } & * \mathrm{p}>\mathrm{p} / \#- \\ \text { para-para } & \text { *paRa } & \text { paha } & \\ \text { pare, paria } & \text { *paria? } & \text { papare } & \\ \text { stingray } & \text { *paRi } & \text { pahi } & \\ \text { how (many) } & \text { *pija } & \text { pila? } & \\ \text { ten } & \text { *pulu? } & \text { polo? } & * \mathrm{p}>\mathrm{p} / \#-\# \\ \text { tipis } & \text { *tipis } & \text { nipi? } & \\ \text { centipede } & \text { *Sipan } & \text { lipan } & \\ \text { four } & \text { *Sopat } & \text { pa? } & \\ \text { what } & \text { *apa } & \text { sapan } & \end{array}$

B. $P A N * t$

$\mathrm{PAN} * \mathrm{t}$ retains regularly only in inter-vocal positions, whereas in other positions are irregular, both retention and innovation

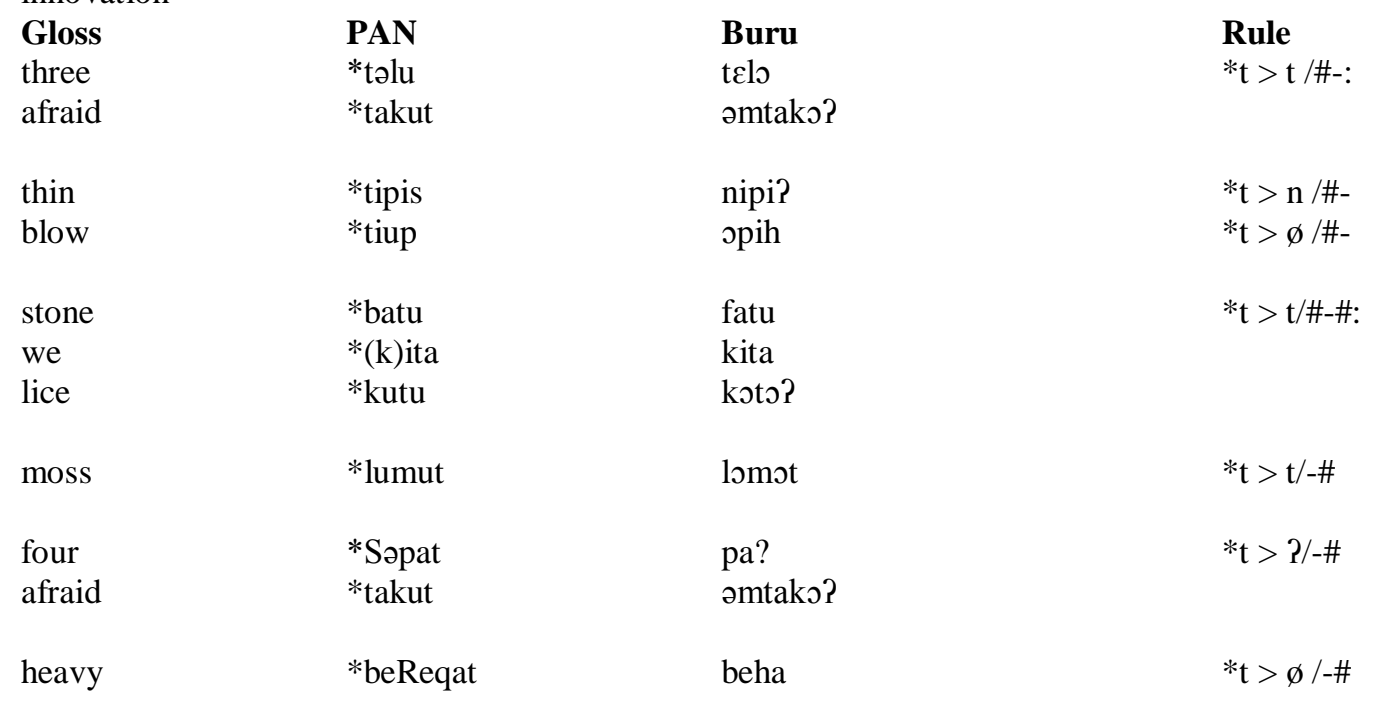

C. $P A N * C$

PAN $* \mathrm{C}$ changes to $/ \mathrm{t} / \mathrm{at}$ the initial and middle position occur regularly, whereas at end position becomes irregular. Besides /t/, PAN $* \mathrm{C}$ also changes to / $\varnothing /$ irregularly at the beginning position.

$\begin{array}{llll}\text { Gloss } & \text { PAN } & \text { Buru } & \text { Rule } \\ \text { know } & * \text { Caqu } & \text { tewa } & * \mathrm{C}>\mathrm{t} / \#- \\ \text { cry } & * \text { Cayis } & \text { taji } & \\ \text { feces } & * \text { Ca?i } & \text { stei } & \\ \text { ear } & * \text { Calina } & \text { linan } & * \mathrm{C}>\emptyset / \#-\end{array}$




$\begin{array}{llll}\text { egg } & \text { *qiColuR } & \text { telun } & * \mathrm{C}>\mathrm{t} / \text { \#-\# } \\ \text { die } & \text { *maCay } & \text { mata? } & \\ \text { octopus } & \text { kuRiCa } & \text { kita? } & \\ & & & \\ \text { vein } & \text { *huRaC } & \text { uhat } & * \mathrm{t} / \text {-\# } \\ \text { sky } & \text { *layiC } & \text { lanit } & \end{array}$

D. $P A N * k$

At the initial and middle position, PAN * $\mathrm{k}$ retains regularly, while at the end, changes to $/ \mathrm{t} / \mathrm{and} / \mathrm{n} / \mathrm{irregularly}$.

\section{Gloss}

octpos

dig

we

lice

eat

open

afraid

fish

child

navel

\section{PAN}

*kuRiCa

*kali

*(k)ita

*kutu

*kaən

*huka?

*takut

*Sikan

*aNak

*pujək

\section{Buru}

kita?

kali

kita

koto?

$\mathrm{ka}$ ?

fukah

əmtako?

ikan

anat

pusen
Rule

*k > k/\#-:

E. PAN *?

PAN *? at the initial changes to / $/$ regularly, while $/ 1, \mathrm{~m}, \mathrm{k} /$ are irregularly. At the middle, PAN *? becomes /w/ irregularly. At the end position, retention and innovation are irregularly (become $/ \mathrm{h}, \mathrm{n}, \mathrm{k}, \mathrm{dan} \phi /$ ).

\begin{tabular}{|c|c|c|c|}
\hline Gloss & PAN & Buru & Rule \\
\hline salt & * PasiRa & sasi & $* ?>\varnothing / \#-$ \\
\hline egg & *2iColur & telun & \\
\hline sand & *Pənay & عna? & \\
\hline pillow & *?alunan & kanluya & $* ?>\mathrm{k} / \#-$ \\
\hline ash & *?abu & lafu & $* ?>1 / \#-$ \\
\hline salty & * Pasin & masin & *? > m/\#- \\
\hline know & *Ca?u & tewa & $* ?>$ w/\#-\# \\
\hline open & *huka? & fukah & $* ?>\mathrm{h} /-\#$ \\
\hline red & *məRa? & merah & \\
\hline ten & *sa-pulu? & polo? & *? > ?/-\# \\
\hline fast & *zulu? & leuk & $* ?>\mathrm{k} /-\#$ \\
\hline blood & *daRa? & rahan & $* ?>n /-\#$ \\
\hline pare & *paria? & papare & $* ?>\varnothing /-\#$ \\
\hline
\end{tabular}

F. $P A N * b$

PAN *b retains only at the initial position in irregular behavior. In Buru, PAN *b tends to innovate into /f/ regular at initial whereas at the middle could be regular if the data are broaden.

$\begin{array}{llll}\text { Gloss } & \text { PAN } & \text { Ambelau } & \text { Rule } \\ \text { new } & \text { *ba?əRuh } & \text { fehu } & * \mathrm{~b}>\mathrm{f} / \#- \\ \text { split } & \text { *bəla? } & \text { fola } & \\ \text { stone } & \text { *batu } & \text { fatu } & \\ \text { moon } & \text { *bulaN } & \text { fulan } & \\ \text { fur } & \text { *bulu } & \text { folon } & \\ \text { pig } & \text { *babuy } & \text { fafu } & \end{array}$




$\begin{array}{llll}\text { weight } & \text { *bəRəPat } & \text { beha } & * \mathrm{~b}>\mathrm{b} / \#- \\ \text { ash } & \text { *Pabu } & \text { lafu } & * \mathrm{~b}>\mathrm{f} / \#-\# \\ \text { pig } & \text { *babuy } & \text { fafu } & \end{array}$

G. $P A N * d$

PAN $* \mathrm{~d}$ tends into $/ \mathrm{r} /$ at the initial or middle position but irregularly.

$\begin{array}{llll}\begin{array}{l}\text { Gloss } \\ \text { blood }\end{array} & \text { PAN } & \text { Buru } & \text { Rule } \\ \text { two } & * \text { daRa? } & \begin{array}{l}\text { rahan } \\ \text { rua }\end{array} & * \mathrm{~d}>\mathrm{r} / \#- \\ \text { they } & \text { *siida } & \text { ira } & * \mathrm{~d}>\mathrm{r} / \#-\# \text { : }\end{array}$

H. $P A N *_{z}, *_{j}$, and $*_{c}$

At initial position PAN *z changes to $/ 1 /$ and $/ \varnothing /$ irregularly. PAN $* \mathrm{j}$, which innovates into $/ \mathrm{s}, 1, \varnothing /$ or retains /j/ is irregularly. PAN *g, found in one datum, retains irregularly at the middle position.

\begin{tabular}{|c|c|c|c|}
\hline Gloss & PAN & Ambelau & Rule \\
\hline walk & *zalan & tolon & $*_{\mathrm{Z}}>\mathrm{t} / \#-$ \\
\hline past & *zulu? & leuk & $*_{\mathrm{Z}}>\emptyset / \#-$ \\
\hline keep & *jagah & jaga & $* \mathrm{j}>\mathrm{j} / \#-$ \\
\hline name & * yajan & yan & $* \mathrm{j}>\emptyset / \#-\#$ \\
\hline navel & *pujək & pusen & $* \mathrm{j}>\mathrm{s} / \#-\#$ \\
\hline some & *pija & pila? & $* \mathrm{j}>1 / \#-\#$ \\
\hline keep & * jagah & jaga & $* \mathrm{~g}>\mathrm{g} / \#-\#$ \\
\hline
\end{tabular}

I. $\quad P A N * m, * n, *_{n}, *_{\eta}$, and $* N$

PAN *m has retention in Buru, irregular at initial position but irregular at the end. At the end, however changes to / $/$ irregularly.

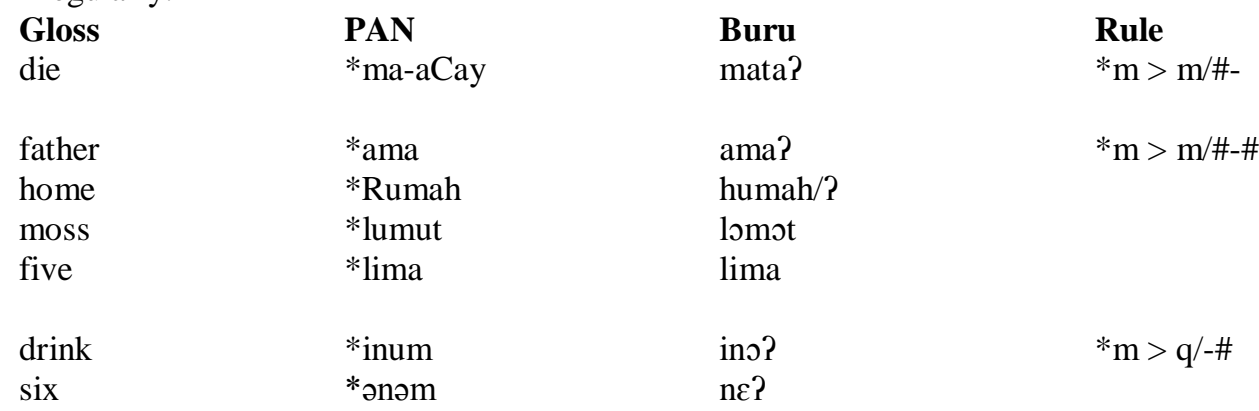

PAN *n in the middle and end still retains regularly Buru. But, PAN *n has innovation at the middle $(/ \mathrm{y} /$ and $/ \mathrm{d} /)$ and at end position (/ø/dan/?/). All are irregular.

\begin{tabular}{|c|c|c|c|}
\hline Gloss & PAN & Buru & Rule \\
\hline mother & $*$ ina & inan & $* \mathrm{n}>\mathrm{n} / \#-\#$ \\
\hline right & ${ }^{*}$ wanaN, *waNan & wanna? & \\
\hline drink & $*$ inum & ino? & \\
\hline sand & *?enay & Ena? & \\
\hline $\operatorname{six}$ & *ənəm & ne? & \\
\hline pillow & *?alunan & kanluya & $*_{n}>\mathrm{y} / \#-\#$ \\
\hline where & $*$ inu & fido & $* \mathrm{n}>\mathrm{d} / \#-\#$ \\
\hline fish & *Sikan & ikan & $*_{\mathrm{n}}>\mathrm{n} /-\#$ \\
\hline name & *yajan & yan & \\
\hline centipede & *Sipan & lipan & \\
\hline salty & *Pasin & masin & \\
\hline
\end{tabular}




\begin{tabular}{|c|c|c|}
\hline pillow & *?alunan & kanluna \\
\hline right & ${ }^{*}$ wanaN, *waNan & wanna? \\
\hline eat & *kaen & ka? \\
\hline
\end{tabular}

PAN $* n$ innovates into $/ \mathrm{n} /$ at the initial. PAN $*_{\eta}$ has irregular retension at middle and end. There is also changes of PAN $*_{\mathrm{y}}$ to $/ \mathrm{n}$ at the middle position irregularly.

\begin{tabular}{|c|c|c|c|}
\hline Gloss & PAN & Buru & Rule \\
\hline coconut & *niuR & niwe? & $*_{\mathrm{n}}>\mathrm{n} / \#-$ \\
\hline name & *yajan & yan & $* \eta>y / \#-:$ \\
\hline sky & *layiC & layit & $* \eta>n / \#-\#:$ \\
\hline ear & *Calina & linan & \\
\hline wind & *hayin & anin & \\
\hline cry & *Cayis & $\operatorname{tani}$ & \\
\hline wind & *hayin & anin & $*_{\mathrm{y}}>\mathrm{n} / \#-\#:$ \\
\hline
\end{tabular}

PAN $* \mathrm{~N}$, at the middle or end postion has irregular innovation.

\begin{tabular}{|c|c|c|c|}
\hline Gloss & PAN & Buru & Rule \\
\hline child & *aNak & anat & $* \mathrm{~N}>\mathrm{n} / \#-\#$ \\
\hline right & ${ }^{*}$ wanaN, ${ }^{*}$ waNan & wanna? & \\
\hline white & $*^{*}$ ma-puNi & boti & $* \mathrm{~N}>\mathrm{t} / \mathrm{H}-\#$ \\
\hline moon & $*$ bulaN & fulan & $* \mathrm{~N}>\mathrm{n} /-\#$ \\
\hline right & *waNan, wanaN & wanna? & $* \mathrm{~N}>$ ?/-\# \\
\hline
\end{tabular}

J. $\quad P A N * S, * s$, dan *h

Phonem *S is omitted at initial and middle position, regular and irregularly. Moreover, PAN *S changes irregularly to /1/ at the middle position.

$\begin{array}{llll}\begin{array}{l}\text { Gloss } \\ \text { meat }\end{array} & \text { PAN } & \text { Buru } & \text { Rule } \\ \text { four } & \text { *Səsi } & \text { isit } & * \mathrm{~S}>\emptyset / \#-\text { : } \\ \text { fish } & \text { *Səpat } & \text { pa? } & \\ \text { *Sikan } & \text { ikan } & \\ \text { centipede } & * \text { Sipan } & \text { lipan } & * \mathrm{~S}>1 / \#- \\ \text { water } & \text { *waSiR } & \text { wae } & * \mathrm{~S}>\emptyset / \#-\# \\ \text { two } & * \text { duSa } & \text { rua } & \end{array}$

Reflex of PAN *s retains both at initial and middle position, but regular at initial and irregular at the middle. At initial and end position, PAN *s innovates $(/ \varnothing /$ dan $/ \Upsilon /)$ and both are irregular.

\begin{tabular}{|c|c|c|c|}
\hline Gloss & PAN & Buru & Rule \\
\hline $\operatorname{dog}$ & $*$ asu & asu & $*_{\mathrm{S}}>\mathrm{s} / \#-$ \\
\hline milk & *susu & sosən & \\
\hline ten & *pulu? & polo? & $*_{\mathrm{S}}>\varnothing / \#-$ \\
\hline meat & $*_{\text {səsi }}$ & isit & $*_{\mathrm{s}}>\mathrm{s} / \#-\#$ \\
\hline milk & *susu & soson & \\
\hline salty & *?asin & masin & \\
\hline cry & *Canis & $\tan \mathrm{i}$ & $*_{\mathrm{s}}>\varnothing /-\#$ \\
\hline thin & *tipis & nipi? & $*_{s}>$ ?/-\# \\
\hline
\end{tabular}


$\mathrm{PAN} * \mathrm{~h}$ is kept irregularly in final position in Buru language. Likewise, the innovations (/ $\varnothing /$ and / $\mathrm{P} / \mathrm{)}$ in the starting and ending positions are irregular

$\begin{array}{llll}\begin{array}{l}\text { Gloss } \\ \text { wind }\end{array} & \text { PAN } & \text { Buru } & \text { Rule } \\ \text { open } & \text { *hayin } & \text { anin } & \text { fh }>\varnothing / \#- \\ \text { vein } & \text { *huka? } & \text { fukah } & \\ & \text { uhat } & \\ \text { house } & \text { *Rumah } & \text { huma(h,? } & * \mathrm{~h}>\mathrm{h} / \text {-\# } \\ \text { new } & \text { *baPəRuh } & \text { fehu } & * \mathrm{~h}>\varnothing / \text {-\# } \\ \text { keep } & \text { *jagah } & \text { jaga } & \\ \text { house } & \text { *Rumah } & \text { huma(h,? } & * \mathrm{~h}>\mathrm{q} / \text {-\# }\end{array}$

K. $P A N * l, * R$, dan *r

PAN * 1 retains regularly in the initial and middle position in Buru. In addition, in the middle position PAN $* 1$ undergoes an irregular innovation into / $\mathrm{r} /$.

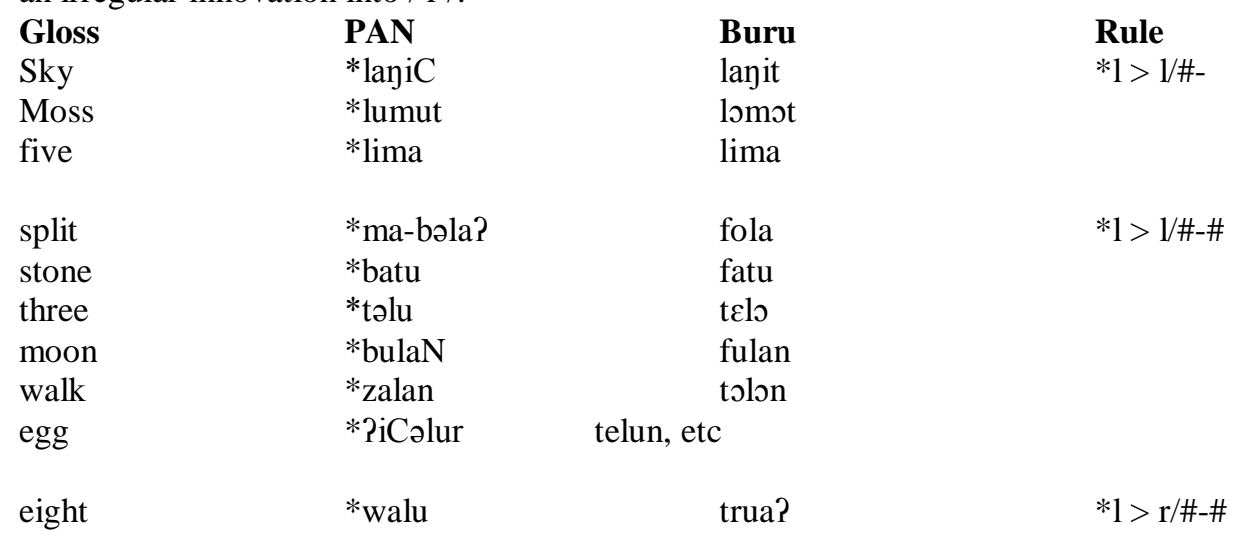

$\mathrm{PAN} * \mathrm{R}$ in the middle position changes to / $\mathrm{h} /$ regularly, while in the initial position is possible to occur regularly if the data are expanded. Besides / h /, PAN $* \mathrm{R}$ changes to / $\mathrm{n} /$ and / $\mathrm{P} /$ in the final position and becomes / $\varnothing /$ (in the middle and end position) and / $\mathrm{s} /$ (in the middle position) each of which is irregular.

\begin{tabular}{|c|c|c|c|}
\hline $\begin{array}{l}\text { Gloss } \\
\text { big } \\
\text { house }\end{array}$ & $\begin{array}{l}\text { PAN } \\
* \text { Raya } \\
* \text { Rumah }\end{array}$ & $\begin{array}{l}\text { Buru } \\
\text { hat } \\
\text { huma }(h, ?)\end{array}$ & $\begin{array}{l}\text { Rule } \\
* \mathrm{R}>\mathrm{h} / \#-\end{array}$ \\
\hline $\begin{array}{l}\text { new } \\
\text { weight } \\
\text { blood } \\
\text { vein } \\
\text { para-para } \\
\text { stingray }\end{array}$ & $\begin{array}{l}\text { *ba?eRuh } \\
\text { *beRəqat } \\
\text { *daRa? } \\
\text { *huRaC } \\
\text { *paRa } \\
\text { *paRi }\end{array}$ & $\begin{array}{l}\text { fehu } \\
\text { beha } \\
\text { rahan } \\
\text { uhat } \\
\text { paha } \\
\text { pahi, etc }\end{array}$ & $* \mathrm{R}>\mathrm{h} / \#-\#$ \\
\hline $\begin{array}{l}\text { octopus } \\
\text { salt } \\
\text { water } \\
\text { tail } \\
\text { coconut }\end{array}$ & $\begin{array}{l}\text { *kuRiCa } \\
\text { *?asiRa } \\
\text { *waSiR } \\
\text { *ikuR } \\
\text { *niuR }\end{array}$ & $\begin{array}{l}\text { kita? } \\
\text { sasi } \\
\text { wae } \\
\text { ikun } \\
\text { niwe? }\end{array}$ & $\begin{array}{l}* \mathrm{R}>\varnothing / \#-\# \\
* \mathrm{R}>\mathrm{s} / \#-\# \\
* \mathrm{R}>\emptyset /-\# \\
* \mathrm{R}>\mathrm{n} /-\# \\
* \mathrm{R}>\mathrm{s} /-\#\end{array}$ \\
\hline
\end{tabular}

PAN * $\mathrm{r}$ has retention in the middle position and innovation $(/ \mathrm{n} /)$ at the end position, each of which is irregular.

$\begin{array}{llll}\text { Gloss } & \text { PAN } & \text { Buru } & \text { Rule } \\ \text { pare } & \text { *paria? } & \text { papare } & * \mathrm{r}>\mathrm{r} / \#-\# \text { : } \\ \text { egg } & \text { *2iColur } & \text { telun } & * \mathrm{r}>\mathrm{n} / \text {-\# }\end{array}$

L. $P A N * w$

$\mathrm{PAN} * \mathrm{w}$ retains irregular in the initial position, while in the middle position becomes / y / irregularly. 


\begin{tabular}{|c|c|c|c|}
\hline Gloss & PAN & Buru & Rule \\
\hline water & *waSiR & waع & $*_{\mathrm{w}}>\mathrm{w} / \mathrm{\#}-$ \\
\hline right & ${ }^{*}$ wanaN, *waNan & wanna? & \\
\hline nine & *siwa & siya? & $*_{\mathrm{w}}>\mathrm{y} / \mathrm{\#}-\mathrm{\#}$ \\
\hline
\end{tabular}

M. PAN *i

PAN * $\mathrm{i}$ has regular retention at the initial position, penultimate syllable, and ultimate syllable, while at the end position is irregular. In addition, PAN * i undergoes irregular innovations at the penultimate syllable $(/$ e $/$ and / $\varnothing /)$ and the ultimate syllable $(/ \varepsilon /)$.

\begin{tabular}{|c|c|c|c|}
\hline Gloss & PAN & Buru & Rule \\
\hline mother & *ina & inan & $* \mathrm{i}>\mathrm{i} / \#-$ \\
\hline we & $*(\mathrm{k})$ ita & kita & \\
\hline drink & *inum & ino? & \\
\hline thin & *tipis & nipi? & $* \mathrm{i}>\mathrm{i} / \# \mathrm{~K}-$ \\
\hline how (many) & *pija & pila? & \\
\hline ear & *Calina & linan & \\
\hline coconut & *niuR & niwe?, etc & \\
\hline pare & *paria? & papare & $* \mathrm{i}>\mathrm{e} / \# \mathrm{~K}-$ \\
\hline egg & *qiCəlur & telun & $* \mathrm{i}>\varnothing / \# \mathrm{~K}-:$ \\
\hline wind & *hayin & anin & $*_{\mathrm{i}}>\mathrm{i} /-\mathrm{K} \#$ \\
\hline sky & *layiC & layit & \\
\hline thin & *tipis & nipi?, etc & \\
\hline water & *waSiR & wae & $* \mathrm{i}>\varepsilon /-\mathrm{K} \#$ \\
\hline stingray & *paRi & pahi & $*_{\mathrm{i}}>\mathrm{i} /-\#$ \\
\hline
\end{tabular}

N. PAN *u

PAN * $\mathrm{u}$ in penultimate syllable, ultimate syllable, and end positions are retained regularly in Buru. In addition, in the penultimate syllable, PAN * $\mathrm{u}$ innovates to / $\mathrm{o}, \rho, \varnothing /$ irregularly. In the ultimate syllable, PAN * u also innovates to / $\rho$, e / irregularly. In the final position, PAN * u innovates to / 0 , a / irregularly.

\begin{tabular}{|c|c|c|c|}
\hline Gloss & PAN & Buru & Rule \\
\hline moon & *bulaN & fulan & $* \mathrm{u}>\mathrm{u} / \# \mathrm{~K}-$ \\
\hline house & * Rumah & huma(h,?) & \\
\hline open & *huka? & fukah & \\
\hline navel & *pujək & pusen & \\
\hline two & $* d u S a$ & rua, etc & \\
\hline fur & *bulu & folon & $* \mathrm{u}>\mathrm{s} / \# \mathrm{~K}-$ \\
\hline milk & $*$ susu & soson & \\
\hline ten & *pulu? & polo? & \\
\hline moss & *lumut & lomot, etc & \\
\hline octopus & *kuRiCa & kita? & $* \mathrm{u}>\emptyset / \# \mathrm{~K}-$ \\
\hline past & $*$ zuluq & leuk & \\
\hline white & *puNi & boti & $* \mathrm{u}>\mathrm{o} / \# \mathrm{~K}-$ \\
\hline new & *baPeRuh & fehu & $* \mathrm{u}>\mathrm{u} /-\mathrm{K} \#$ \\
\hline egg & *?iColur & telun & \\
\hline past & $*_{\text {zulu? }}$ & leuk & \\
\hline drink & *inum & ino? & $* \mathrm{u}>\mathrm{s} / \mathrm{-K} \#$ \\
\hline
\end{tabular}




\begin{tabular}{|c|c|c|c|}
\hline $\begin{array}{l}\text { afraid } \\
\text { moss } \\
\text { ten }\end{array}$ & $\begin{array}{l}\text { *takut } \\
* \text { lumut } \\
* \text { pulu? }\end{array}$ & $\begin{array}{l}\text { omtako? } \\
\text { lomot } \\
\text { polo?, etc }\end{array}$ & \\
\hline coconut & *niuR & niwe? & $*_{\mathrm{u}}>\mathrm{e} / \mathrm{-K} \#:$ \\
\hline $\begin{array}{l}\text { ash } \\
\text { stone } \\
\text { dog }\end{array}$ & $\begin{array}{l}\text { * Pabu } \\
* \text { batu } \\
* \text { asu }\end{array}$ & $\begin{array}{l}\text { lafu } \\
\text { fatu } \\
\text { asu }\end{array}$ & $*_{\mathrm{u}}>\mathrm{u} /-\#$ \\
\hline three & $*$ təlu & telo & $* u>\rho /-\#$ \\
\hline know & *Ca?u & tewa & $* u>a /-\#$ \\
\hline
\end{tabular}

O. $P A N * \partial$

PAN *ə regularly innovate to /e/ at penultimate syllable. Besides /e/, PAN *ə also changes to /o, i, $\varepsilon /$ at penultimate syllable, and $/ \varepsilon /$ at ultimate syllable, both are irregular.

\begin{tabular}{|c|c|c|c|}
\hline Gloss & PAN & Buru & Rule \\
\hline new & *baPəRuh & fehu & $*_{\partial}>\mathrm{e} / \# \mathrm{~K}-$ \\
\hline weight & *bəRəPat & beha & \\
\hline egg & *?iColur & telun, etc & \\
\hline split & *ma-bəla? & fola & $*_{\partial}>\mathrm{o} / \# \mathrm{~K}-$ \\
\hline meat & *səsi & isit & $*_{\partial}>\mathrm{i} / \# \mathrm{~K}-$ \\
\hline sand & *?ənay & ena? & $*_{\partial}>\varepsilon / \# \mathrm{~K}-$ \\
\hline three & *təlu & telo & \\
\hline navel & *pujək & pusen & $*_{\partial}>\varepsilon /-\mathrm{K} \#$ \\
\hline six & *ənəm & nع? & \\
\hline
\end{tabular}

P. PAN*a

PAN *a has regular retention at initial position, penultimate syllable, ultimate syllable, and at end position. At penultimate syllable PAN *a also irregularly innovate to /e,,$\varnothing /$; at ultimate syllable irregularly innovate to / / and / $/$. Likewise at the end position, PAN *a has irregular innovation to $/ \varepsilon /$ and $/ \varnothing /$.

\begin{tabular}{|c|c|c|c|}
\hline Gloss & PAN & Buru & Rule \\
\hline child & *aNak & anat & $* a>a / \#-$ \\
\hline $\operatorname{dog}$ & *asu & asu & \\
\hline father & *ama & ama?, etc & \\
\hline ash & *Pabu & lafu & $* a>a / \# K-$ \\
\hline water & *waSiR & wae & \\
\hline wind & *hayin & anin & \\
\hline stone & *batu & fatu & \\
\hline stingray & *paRi & pahi, etc & \\
\hline ear & *Calina & linan & $* a>\varnothing / \# \mathrm{~K}-$ \\
\hline know & $*$ Caqu & tewa & $* a>$ e/\#K- \\
\hline walk & *zalan & tolon & $* a>s / \# \mathrm{~K}-$ \\
\hline urat & *huRaC & uhat & $* a>a /-K \#$ \\
\hline how (many) & *pija & pila? & \\
\hline mother & *ina & inan & \\
\hline moon & *bulaN & fulan, etc & \\
\hline lice & *kutu & koto? & $* \mathrm{a}>\mathrm{s} /-\mathrm{K} \#$ \\
\hline walk & *zalan & tolon & \\
\hline
\end{tabular}




\begin{tabular}{|c|c|c|c|}
\hline $\begin{array}{l}\text { name } \\
\text { pare }\end{array}$ & $\begin{array}{l}\text { *yajan } \\
\text { *pariaq }\end{array}$ & $\begin{array}{l}\text { yan } \\
\text { papare }\end{array}$ & $* \mathrm{a}>\varnothing /-\mathrm{K} \#$ \\
\hline $\begin{array}{l}\text { father } \\
\text { we } \\
\text { ear } \\
\text { five } \\
\text { nine }\end{array}$ & $\begin{array}{l}\text { *ama } \\
*(\mathrm{k}) \text { ita } \\
\text { *Calina } \\
\text { *lima } \\
\text { *siwa }\end{array}$ & $\begin{array}{l}\text { ama? } \\
\text { kita } \\
\text { linan } \\
\text { lima } \\
\text { siya?, etc }\end{array}$ & $* a>a /-\#$ \\
\hline $\begin{array}{l}\text { navel } \\
\text { salt }\end{array}$ & $\begin{array}{l}\text { *puja } \\
\text { *qasiRa }\end{array}$ & $\begin{array}{l}\text { pusen } \\
\text { sasi }\end{array}$ & $\begin{array}{l}* a>\varepsilon /-\# \\
* a>\varnothing /-\#\end{array}$ \\
\hline
\end{tabular}

Q. PAN *ay

PAN *ay at the end position innovates irregularly, but could be regular if the data are extended into /a/.

$\begin{array}{llll}\text { Gloss } & \text { PAN } & \text { Buru } & \text { Rule } \\ \text { die } & \text { *ma-aCay } & \text { mata? } & * \text { ay }>\text { a/-\# } \\ \text { sand } & \text { *qenay } & \text { Ena? } & \end{array}$

\section{SOME Notes ON COLLINS (1981) VIEWS}

Historically, Buru language in the first phase split from the Ambelau language and merged with the Sula and Taliabo languages. According to Collins (1981), Buru languages along with Sula and Taliabo, PAN * $\mathrm{t}$ in the initial position (\#-) and the vowel (\# v-v \#) change to /f/. This study shows that PAN * $\mathrm{t}$ at initial and intervocal positions are retained as /t/ regularly. In addition, PAN $* t$ in the initial position becomes $/ \mathrm{n} /$ and $/ \varnothing /$. The result identifies that there is no PAN *t change into /f/ in Buru language. Therefore, the linguistic evidence of the historical relation which separates Buru from Ambelau, and its unity with Sula and Taliabo, which Collins (1981) proposes, needs to be reviewed.

Secondly, PAN: *k /\#- and \#v-v\# in Buru with Sula and Taliabo are retained, while in Ambelau is innovated. The evidence that Collins (1981) proposes, can be justified, since both the initial and inter-vocal positions of PAN * $\mathrm{k}$ have retention in Buru. There is a PAN $*_{\mathrm{k}}$ in the initial position of being innovated (being /n/) but irregular. Likewise in the final position, although innovated to be /t/ but irregular. It means that the linguistic evidence from Collins (1981) can still be used to view of the historical relations of the West Central Maluku languages .

Third, the merger of PAN *k and *p at the end position into /t/ in Buru, Sula and Taliabo. This study shows that no PAN * $\mathrm{p}$ at the final position changes to / $t$ / in Buru, whereas PAN *k becomes /t/ irregularly, for example in the meaning of 'child', PAN $* \mathrm{aNak}>\mathrm{Br}$ : anat. Therefore, no merger of PAN *p and *k in Buru. Thus, such linguistic evidence cannot be used as the basis for viewing the historical relation of West Central Maluku languages which Collins hypothesized (1981).

For PAN *mb changes to /b/ in Buru (also Sula and Taliabo) and merger PAN * $\mathrm{y}$ and * $\mathrm{yk}$ become /g/, further study is needed. The data obtained have not shown the realization of these sounds in Buru language.

\section{CONCLUSION}

The PAN phonemic reflex into Buru language shows the existence of retention and innovation sounds, regular and irregular. There is one evidence that can be used, ie PAN $* \mathrm{k}$ in the initial and middle position. However, PAN *t in the initial and intervocal positions become /f/ and also the PAN merger: *k and *p at the end position to /t/ in Buru, Sula, and Taliabo languages cannot be used to explain the historical relation of languages which belongs to the West Central Maluku Group referred by Collins (1981). For proofs showing that PAN *mb changes to /b/ and the merger of PAN * $*$ and * ${ }^{\mathrm{k}} \mathrm{k}$ to $/ \mathrm{g} /$ in Buru, and Sula and Taliabo need further study.

To explain adequately and comprehensively the Collins hypothesis (1981) on the historical relation of West Central Maluku languages, a study of PAN reflex into the Sula and Talibo languages needs to be carried out after Ambelau and Buru languages have been formulated. In turn, it will appear to be a common form of innovation that occurs in the fourth language. That is, it is necessary to study PAN reflexes into Sula and Taliabo and the historical relations of west Maluku languages thoroughly.

\section{References}

Blust, Robert A. (1980). Early Austronesian Social Organization the Evidence of Language. Current Anthropology, 21(2), $205-266$.

Blust, Robert A. and S. Trussel. (2015). AustronesianComparative Dictionary. Available online at ww.trussel2.com/ACD .

Collins, James T. (1981). Preliminary Notes on Proto-West Central Maluku: Buru, Sula, Taliabo, and Ambelau. Historical Linguistics in Indonesia Part I Journal. Seri NUSA. Jakarta: Universitas Atma Jaya.

Sumarlam, dkk. (2017). Menguji Hipotesis Collins (1981) tentang Keberadaan Kelompok Maluku Tengah Barat. Laporan Penelitian Hibah Pascasarjana 2017 Menristekdikti Tahun Pertama. Surakarta: Universitas Sebelas Maret. 